\title{
New Approach to the Strain Analysis of Bragg Grating Sensors
}

\author{
José R. Alves da CUNHA ${ }^{1 *}$, Petrus Alcantara JR. ${ }^{1}$, Angela Santa BRÍGIDA ${ }^{2}$, \\ Gilvan BORGES ${ }^{2}$, and João Weyl COSTA ${ }^{2}$
}

\author{
${ }^{1}$ Programa de Pós-Graduação em Física, Universidade Federal do Pará, Belém, 66075-110, Brazil \\ ${ }^{2}$ Programa de Pós Graduação em Engenharia Elétrica, Universidade Federal do Pará, Belém, 66075-110, Brazil \\ *Corresponding author: José R. Alves da CUNHA_ E-mail: jrenato@ufpa.br
}

\begin{abstract}
The response of an optical strain sensor based on the fiber Bragg grating is theoretically analyzed with a different approach of that found usually in the literature. In this model, geometrical changes suffered by the grating itself are taken into account. The results are compared with those from experiments, showing very good agreement.
\end{abstract}

Keywords: Bragg grating sensor, strain sensor, Bragg wavelength

Citation: José R. Alves da CUNHA, Petrus Alcantara JR., Angela Santa BRÍGIDA, Gilvan BORGES, and João Weyl COSTA, "New Approach to the Strain Analysis of Bragg Grating Sensors," Photonic Sensors, vol. 3, no. 1, pp. 74-80, 2013.

\section{Introduction}

The technology of optical fiber sensors (OFS) has been quickly developed in the last years [1]. Among several classes of OFS, those based on Bragg gratings are excellent choices for applications where systems sensing more traditional, like electromagnetics, is shown to be inefficient because they are excellent sensors for the measurement of static and dynamic physical quantities such as temperature, strain and pressure [2].

Through time, the fiber Bragg grating sensors (FBGS) were developed and tested in different situations, especially in smart structures [3, 4], showing satisfactory results and high reliability. Compared to their analogues, the FBGS have high sensitivity, immunity to electromagnetic interference, small size, low weight, easy adaptation to work structures and facility in transmission of light. The major advantage is that the information to be measured is contained in the wavelength, making the sensor self referenced. [5].

Usually, the change in the Bragg wavelength is explained by dependency on the gratings parameters with the mechanical strain suffered by the fiber, an effect commonly known as the opto-mechanical effect, in which the photo-elastic effect, the dependence on the refraction index with the mechanical strain, indicates that the variation of the Bragg wavelength varies linearly with the relative strain suffered by the fiber Bragg grating (FBG) and the angular coefficient of the linear function obtained in terms of components of the opto-mechanical tensor [6].

Some researchers studied the effects of temperature and pressure on the refraction index of fused silica and concluded that the influence of temperature on the refraction index was much

Received: 1 August 2012 / Revised version: 15 August 2012

(C) The Author(s) 2012. This article is published with open access at Springerlink.com 
smaller than changes due to variations in the volume (strain, stress, pressure, etc.). Furthermore, methods for interrogating of strain sensors using two identical FBGS allow eliminating the influence of temperature $[7,8]$.

An FBG consists of a periodic modulation of the refraction index in the core of a monomode optical fiber. In this kind of grating, the fronts of the phase are perpendicular to the longitudinal axis of the fiber, and the planes of the grating have a constant period (1). Light guided in the core of an optical fiber will be scattered by each plane of the gratings. If the Bragg condition is satisfied, the light reflected by each plane of the grating is added constructively to form a peak reflected back with a wavelength defined by the parameters of the grating, giving us (1) [6]:

$$
\lambda_{B}=2 n_{\mathrm{ef}} \Lambda
$$

where the Bragg wavelength, $\lambda_{B}$, is the central wavelength of the incident light which is reflected in the Bragg grating, $n_{\text {ef }}$ is the effective refraction index of the core of the fiber in the wavelength. When the FBG suffers external stimuli, such as the strain or a change in temperature, both $n_{\mathrm{ef}}$ and $\Lambda$ may vary making the wavelength also to be varied. This allows that the Bragg grating can be used as a sensor element to vary any stimulus of $n_{\text {ef }}$ and $\Lambda$, such as mechanical stress, temperature, pressure and others. The effect of the strain above can be expressed as $[9,10]$

$$
\Delta \lambda_{B}=\lambda_{B}\left(1-p_{e}\right) \varepsilon_{L}
$$

where $\varepsilon_{L}$ is the relative longitudinal strain suffered by the fiber $\left(\varepsilon_{L}=\Delta L / L\right), \Delta L$ is the variation of the fiber length, $L$ is the fiber length without the strain, and $p_{e}$ is an effective photoelastic constant defined as

$$
p_{e}=n_{\mathrm{ef}}^{2} / 2\left[p_{11}-v\left(p_{11}+p_{12}\right)\right]
$$

where $p_{11}$ and $p_{12}$ are components of the photoelastic tensor with typical values $p_{11}=0.113$ and $p_{12}=0.252$ for the silica fiber doped with germanium. The symbol $v$ is the Poisson coefficient with the typical value of $v=0.16, n_{\mathrm{ef}}=1.482$ [6].
Equation (2) shows that the change in the Bragg wavelength of a strain sensor is linearly proportional to the magnitude of the strain. That ensures an important feature of this sensor: its linearity with the applied stimulus. Using the values of $p_{11}, p_{12}, n_{\mathrm{ef}}$ and $\Lambda$, it is estimated that an optical sensor of the strain FBG can have a sensitivity, calculated with (2) and (3) of approximately $1.2 \mathrm{pm} / \mu \varepsilon$ to the FBG with a highest reflectivity at $1550 \mathrm{~nm}$ [11].

\section{Alternative analysis}

In the usual model, the profile of the refraction index of an FBG depends on extrinsic quantities on the fiber such as the temperature and strain. This property allows Bragg gratings to be used as the sensors, which can be measured by processing the signal reflected by the grating. Based on this, our model presented a theoretical analysis of the optical properties of a Bragg grating acting as a strain sensor. In this analysis, an FBG optical sensor with a length $L_{0}$, diameter $d_{0}$ and the volume $V_{0}$, would be submitted to a strain in the longitudinal direction $\left(\varepsilon_{L}\right)$, which caused a variation in the length $(\Delta L)$, diameter $(\Delta d)$ and consequently in the final volume $\left(V_{f}\right)$ of the fiber, as shown in Fig. 1.

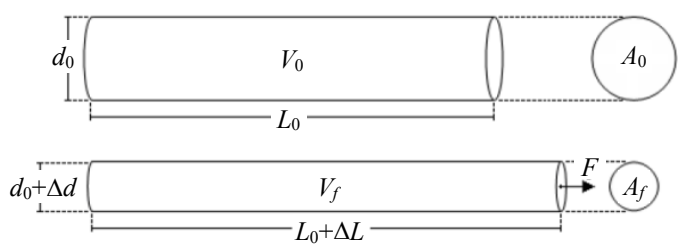

Fig. 1 Core of an optical fiber submitted to an axial traction.

Since this is a cylindrical fiber, which has a cross section of the well-defined area, when it is stretched along the axis, the fiber also suffers a transversal strain $\left(\varepsilon_{t}\right)$. Furthermore, these variations in the diameter and length of the fiber theoretically allow to control the structure of the strain to be measured, because from the longitudinal and transverse displacements, the final length of the fiber $\left(L_{f}\right)$ and its final area $\left(A_{f}\right)$ are calculated, so the volume of the fiber changes, thus allowing the construction of the 
volume of the fiber as a function of the stimulus applied on the $z$ axis, as expressed by (4)

$$
V\left(\varepsilon_{L}\right)=L\left(\varepsilon_{L}\right) A\left(\varepsilon_{L}\right) \rightarrow V\left(\varepsilon_{L}\right)=\left(1+\varepsilon_{L}\right)\left(1-v \varepsilon_{L}\right)^{2} V_{0}
$$

where $L\left(\varepsilon_{L}\right)$ and $A\left(\varepsilon_{L}\right)$ represent the length and area after experiencing the strain. Note that from now on only a longitudinal strain will be used, since the transversal strain will be given as a function of $\varepsilon_{L}$, using the Poisson coefficient, for small values of $\varepsilon_{L}$ terms of second and third orders can be discarded, then

$$
V\left(\varepsilon_{L}\right)=\left[1+(1-2 v) \varepsilon_{L}\right] V_{0} .
$$

Equation (5) determines the volumetric behavior of a body submitted to the small longitudinal strain. This approach is different from the previous by presenting a higher number of parameters of the grating varying under the action of the measurand.

The number of electric dipoles in a volume element of the fiber as a function of the longitudinal strain is given by

$$
N\left(\varepsilon_{L}\right)=N\left(1-G v \varepsilon_{L}\right)
$$

where $N$ is the number of electric dipoles per unit of the volume, and $G$ is a constant related to the density of dipoles.

It is able to get the refraction index as a function of the strain and initial refraction index of the fiber $n(0)$ by the equation

$$
n\left(\varepsilon_{L}\right)=\frac{\sqrt{n(0)^{2}\left(1-G v \varepsilon_{L}\right)+[1-(2-G) v] \varepsilon_{L}}}{1+(1-2 v) \varepsilon_{L}} .
$$

Equation (7) determines the behavior of the refraction index of the FGB submitted to the longitudinal strain. Consider a uniform Bragg grating inscribed in an optical fiber with the refraction index $n_{\text {ef. }}$. The profile of modulation of the refraction index can be expressed as [1]

$$
n_{0}(z)=n_{\mathrm{ef}_{0}}+\Delta n_{0} \cos \left(\frac{2 \pi}{\Lambda_{0}} z\right)
$$

where $\Delta n_{0}$ is the magnitude of the index perturbation, $n_{\mathrm{ef}}$ is the effective refraction index of the core, and $\Lambda_{0}$ is the periodicity of the grating, all them without strain; $z$ is the distance along the longitudinal axis of the fiber, and $\omega=2 \pi / \Lambda_{0}$ is the angular frequency.

Using the profile of modulation of the refraction index shown in (8) and expanding (7) in a binomial series, it has

$$
\begin{aligned}
& n\left(\varepsilon_{L}\right)=\frac{\sqrt{{\mathrm{ef}_{0}}^{2}\left(1-G v \varepsilon_{L}\right)+[1-(2-G) v] \varepsilon_{L}}}{1+(1-2 v) \varepsilon_{L}} \\
& +\frac{\left(1-G v \varepsilon_{L}\right) n_{\mathrm{ef}_{0}} \Delta n_{0} \cos \omega z}{\sqrt{n_{\mathrm{ef}_{0}}^{2}\left(1-G v \varepsilon_{L}\right)+[1-(2-G) v] \varepsilon_{L} \cdot\left[1+(1-2 v) \varepsilon_{L}\right]}} .
\end{aligned}
$$

Physically, as a consequence of variation in the size of the grating in its axial dimension, there is a change of the scale in the profile of the magnitude of the index perturbation in the same direction ( $z$-axis). A longitudinal strain in the fiber causes an increase both in the length of the grid and in the period of modulation. In other words, these quantities will be functions of the longitudinal strain.

$$
\Lambda\left(\varepsilon_{L}\right)=\left(1+\varepsilon_{L}\right) \Lambda_{0} .
$$

Then, the refraction indexes considering the change of the scale in the $z$ axis and the angular frequency are

$$
\begin{aligned}
n\left(\varepsilon_{L}\right)= & \frac{\sqrt{{\mathrm{ef}_{0}}^{2}\left(1-G v \varepsilon_{L}\right)+[1-(2-G) v] \varepsilon_{L}}}{1+(1-2 v) \varepsilon_{L}} \\
& +\frac{\left(1-G v \varepsilon_{L}\right) n_{\mathrm{ef}_{0}} \Delta n_{0}}{\sqrt{n_{\mathrm{ef}_{0}}^{2}\left(1-G v \varepsilon_{L}\right)+[1-(2-G) v] \varepsilon_{L} \cdot\left[1+(1-2 v) \varepsilon_{L}\right]}} \cos \left(\frac{2 \pi z}{\left(1+\varepsilon_{L}\right) \Lambda_{0}}\right),
\end{aligned}
$$

$$
n_{0}\left(\varepsilon_{L}\right)=n_{\mathrm{ef}_{0}}\left(\varepsilon_{L}\right)+\Delta n\left(\varepsilon_{L}\right) \cos \left(\frac{2 \pi}{\Lambda\left(\varepsilon_{L}\right)} z\right) .
$$

As the profile of the refraction index of the FBG is given by (11), it can be compared with (9) to conclude that the effective refraction index $n_{\mathrm{ef}}$ and 
its magnitude of the index perturbation $\Delta n$ depend on the longitudinal strain of the fiber, $\varepsilon_{L}$, of the fiber.

$$
\begin{aligned}
& n_{\mathrm{ef}}\left(\varepsilon_{L}\right)=\sqrt{\frac{n_{\mathrm{ef}_{0}}^{2}\left(1-G v \varepsilon_{L}\right)+[1-(2-G) v] \varepsilon_{L}}{1+(1-2 v) \varepsilon_{L}}},(13) \\
& \Delta n\left(\varepsilon_{L}\right)= \\
& \frac{\left(1-G v \varepsilon_{L}\right) n_{\mathrm{ef}_{0}} \Delta n_{0}}{\sqrt{n_{\mathrm{ef}_{0}}^{2}\left(1-G v \varepsilon_{L}\right)+[1-(2-G) v] \varepsilon_{L}\left[1+(1-2 v) \varepsilon_{L}\right]}} .
\end{aligned}
$$

Equation (11) determines the behavior of the profile of the magnitude of the index perturbation of a uniform Bragg grating submitted to the small longitudinal strain. Equations (10), (13) and (14) show that the period of modulation, the refraction index and the magnitude of the index perturbation of the grating, also vary with the strain applied to the grating.

The variations mentioned above may be used to verify changes produced in the reflectivity spectrum, as well as to develop mathematical models that describe the behavior of certain specific parameters of that spectrum as the Bragg wavelength, maximum reflectivity and bandwidth.

Based on the Bragg condition and the fact that the effective refraction index and the period of the grating are functions of the strain, one can determine the behavior of the Bragg wavelength as

$$
\Delta \lambda_{B}\left(\varepsilon_{L}\right)=\lambda_{B_{0}}\left\{1-\frac{1}{2}[1-(2-G) v]\left(1-\frac{1}{n_{\mathrm{ef}_{0}}^{2}}\right)\right\} \varepsilon_{L} .
$$

The equation above describes the behavior of the Bragg wavelength of a uniform FBG, as a function of the longitudinal strains.

It is also possible to use this model to determine the maximum reflectivity and the bandwidth of a uniform FGB under strain:

$$
R_{\mathrm{MAX}}(\varepsilon)=\tanh ^{2}[k(\varepsilon) L(\varepsilon)]=\tanh ^{2}\left(\frac{\Delta n(\varepsilon) L(\varepsilon) \pi}{2 n_{\mathrm{ef}}(\varepsilon) \Lambda(\varepsilon)}\right)
$$

where

$$
\begin{gathered}
k=\frac{\Delta n \pi}{\lambda_{B}} \\
\Delta \lambda\left(\varepsilon_{L}\right)=\Delta \lambda\left\{1-\frac{1}{2}[1-(2-G) v]\left(1-\frac{1}{n_{\mathrm{ef}_{0}}^{2}}\right)\right\} \varepsilon_{L}+\Delta \lambda_{0} .
\end{gathered}
$$

Equations (16) and (18) describe the linear behavior of the maximum reflectivity and the bandwidth of a uniform FBG, as a function of small longitudinal strains.

\section{Results}

Based on the calculations performed in alternative analysis, one can determine the behavior of the reflectivity of a Bragg grating which has the initial refraction index $n_{\mathrm{ef}}=1.468$, magnitude of the index perturbation $\Delta n=6 \times 10^{-5}$, period $\Lambda=$ $0.531 \mu \mathrm{m}$, and length $L=10 \mathrm{~mm}$. Figure 2 shows the effect of the displacement of the reflectivity spectrum when an FBG is deformed by $200 \mu \varepsilon$ (equivalent to $200 \mu \mathrm{m} / \mathrm{m}$ ) by 10 successive strains of $20 \mathrm{~mm}$ each, based on (16) and considering the dependency of the length of the grating on the strain.

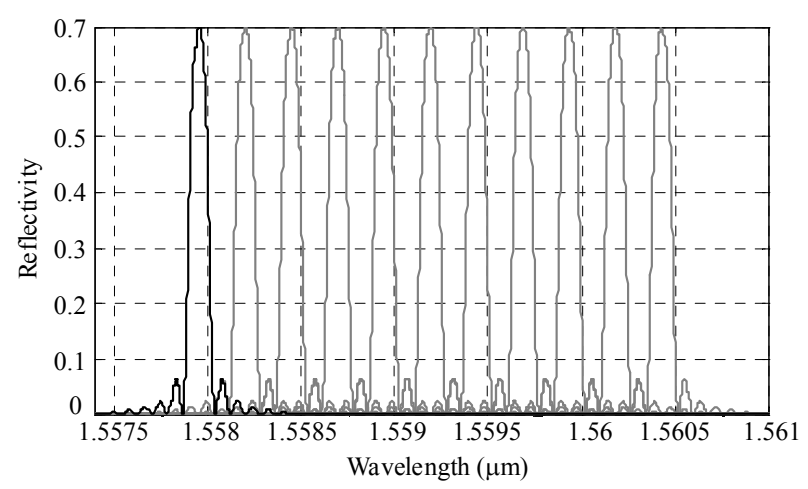

Fig. 2 Reflectivity spectrum of a uniform FBG, submitted to successive strains.

Analyzing Fig. 2, one observes a shift in the reflectivity spectrum after the fiber experiencing the strain and also a small decreasing in the peak of the maximum reflectivity. Therefore, this graph could not be constructed using the usual model currently found, because this model does not discuss the 
dependence of the reflectivity spectrum on the variation of the length of the grating.

Figure 3 shows the behavior of the bandwidth of a uniform FBG considering the same parameters used in Fig. 2, where an increase in the bandwidth can be observed when increasing the strain. This graph also could not be constructed from the usual model by the same reasons of the previous figure.

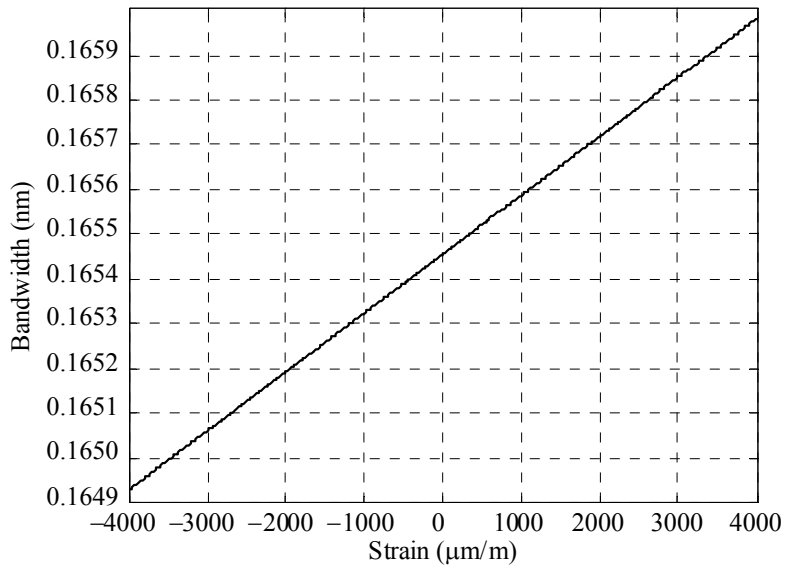

Fig. 3 Behavior of a weak grade increasing with the strain of the FBG.

The analysis was compared with the experimental results $[7,8]$. In the experiments, an FBG was used, provided by MPB Technologies Inc. (serie number: 0503030132011187) with a Bragg wavelength $\lambda_{B}=1553.5 \mathrm{~nm}$ and $n_{\mathrm{ef}}=1.47$. The fiber was strained only along its axis, and the values of the applied tractions were indicated directly by a digital dynamometer. The measurement began when no force acted on the FBG, where a Bragg wavelength for the FBG free from the strain was measured. The FBG was then deformed by the external force applied up to $0.4 \mathrm{kgf}$, for measuring each response of the Bragg wavelength with an optical spectrum analyzer. Using the equation

$$
\frac{F}{A}=E \varepsilon_{L}
$$

where $F$ is the external force strength, $\varepsilon_{L}$ is the longitudinal strain of the fiber, $E$ is the elasticity module for the fiber used, with a value of $72500 \mathrm{~N} / \mathrm{mm}^{2}$, and $A$ is the area of the cross section of the fiber which have a diameter of $125 \mu \mathrm{m}$. It is possible to obtain the strain for each strength value.

Figure 4 shows the result of the combination of (15) which represents the response of the FBG sensor in the present analysis, with the experimental measurements mentioned. The intention was to obtain the value of the $G$ constant for a silica fiber doped with germanium used in the experiment, and it was found $G=0.573$.

It is able to note the linearity of the results obtained in the experiment. These results are consistent with the prediction of the alternative analysis.

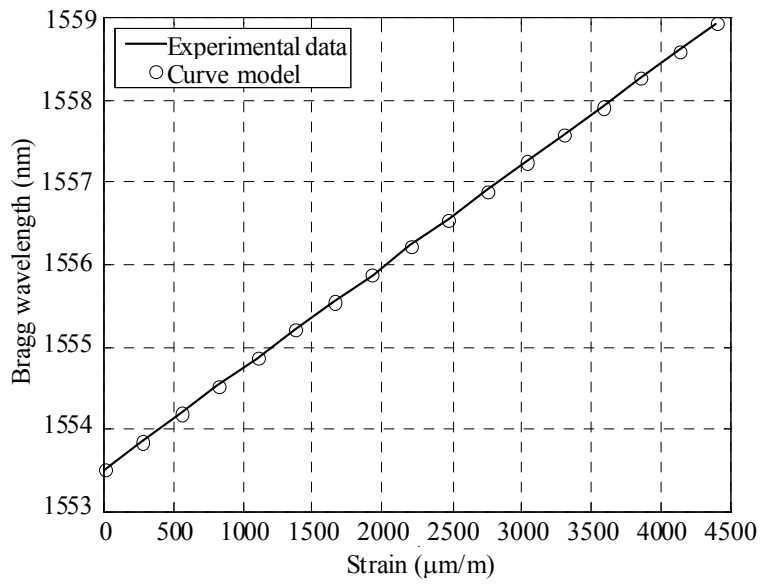

Fig. 4 Theoretical (line) and experimental (circles) behaviors of $\lambda_{B}$ with the strain of an FBG centered on $\lambda_{B 0}=1553.5 \mathrm{~nm}, v=$ 0.16 and $n_{\text {ef }}=1.47$.

In Fig. 5, a comparison between the graphs generated by alternative analysis (15) and the conventional analysis (2) is performed, considering the same FBG specified in Fig. 2, where the usual analysis existing in the literature also describes the behavior of the Bragg wavelength $\left(\lambda_{B}\right)$ as a function of the small longitudinal strain. The error found is only $0.23 \%$.

Comparing the sensitivity for analysis and using the following parameters: $n_{\mathrm{ef}}=1.482, G=0.573, v=$ 0.16 and $\lambda_{B}=1550 \mathrm{~nm}$, it is obtained that the sensitivity found by the usual analysis (2) is $1.2 \mathrm{pm} / \mu \varepsilon$, while by the present analysis (15) it is $1.54 \mathrm{pm} / \mu \varepsilon$. The same sensitivities considering the experimental parameters [7], $n_{\mathrm{ef}}=1.47, G=0.573$, $v=0.16$ and $\lambda_{B}=1553.5 \mathrm{~nm}$, are $1.22 \mathrm{pm} / \mu \varepsilon$, by the 
usual analysis and $1.23 \mathrm{pm} / \mu \varepsilon$, by the analysis proposed in this work.

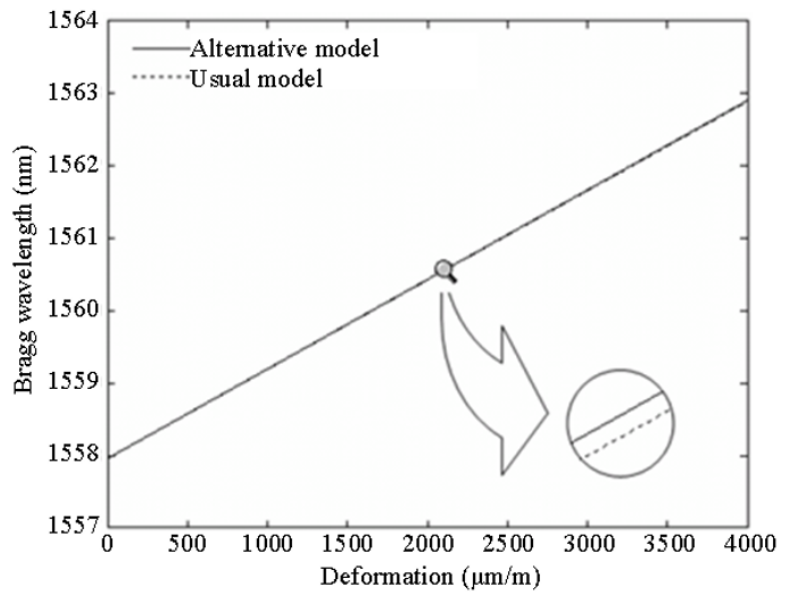

Fig. 5 Comparison between the usual and the present analyses for the FBG optical sensor signal.

\section{Conclusions}

In this paper, a theoretical approach to the behavior of a strain sensor using an FBG is presented, describing the explicit dependence of the profile of the refraction index of the grating, of the reflectivity of the grating, of the bandwidth and of the variation of the Bragg wavelength, on the relative longitudinal strain. In other words, this model is a new analysis that describes the changes in the parameters mentioned.

To obtain these parameters, a theoretical analysis of the optical properties of an FBG as acting as a strain sensor was performed. In this analysis, the fiber was submitted to a longitudinal strain causing a change in the final volume of the fiber which was calculated in the function of their strain. Based on this calculation, the argument that after the longitudinal strain of the fiber the number of electric dipoles in any volume element varied, but the number of dipoles per unit volume of the whole FBG remained constant to arrive in this alternative analysis, was introduced.

Based on the new analysis, the behavior of the Bragg wavelength as a function of small strains, a comparison with experimental results obtained $[7,8]$ was made, as well as with existing results in the literature and also with other experimental results, always resulting in a good degree of concordance.

The proposed alternative analysis stands out because it is more general, since it describes the entire spectrum of reflectivity of the grating, not only certain specific parameters of the spectrum, as it does, the analysis was found in the literature.

It is also able to verify the mathematical method presented for obtaining a new theoretical analysis not using the components of the photoelastic tensor that are used to obtain the existing analysis in the literature.

\section{Acknowledgment}

The authors thank for the financial support of ELETRONORTE, Brazil.

Open Access: This article is distributed under the terms of the Creative Commons Attribution License which permits any use, distribution, and reproduction in any medium, provided the original author(s) and source are credited.

\section{References}

[1] H. J. Patrick, G. M. Williams, A. D. Kersey, J. R. Pedrazzani, and A. M. Vengsarkar, "Hybrid fiber Bragg grating long period fiber grating sensor for strain/temperature discrimination," IEEE Photonics Technology Letters, vol. 8, no. 9, pp. 1223-1225, 1996.

[2] A. Selvarajan, "Fiber optic sensors and their applications," Indian institute of science, Bangalore-560 012, 2010.

[3] Z. Zhou, T. W. Graver, L. Hsu, and J. Ou, "Techniques of advanced FBG sensors: fabrication, demodulation, encapsulation and their application in the structural health monitoring of bridges," Pacific Science Review, vol. 5, no. 1, pp.116-121, 2003.

[4] W. W. Morey, G. Meltz, and W. H. Glenn, "Fiber optic Bragg grating sensors," in Proc. SPIE, vol. 1169, pp. 98-107, 1989. 
[5] E. J. Friebele, "Fiber Bragg grating strain sensors: present and future applicationsin smart structures," Optics and Photonics News, vol. 9, no. 8, pp. 33-37, 1998.

[6] A. Othonos, "Fiber Bragg gratings," Review of Scientific Instruments, vol. 68, no. 12, pp. 4309-4341, 1997.

[7] G. Gagliardi, M. Salza, P. Ferraro, and P. D. Natale, "Interrogation of FBG-based strain sensors by means of laser radio-frequency modulation techniques," Journal of Optics A: Pure and Applied Optics, vol. 8, no. 7, pp. S507-S513, 2006.

[8] C. Floridia, A. C. Santa Brígida, F. Borin, D. C. Dini, P. Alcantara Jr., and N. Bramatti, "Single-axis fiber optic proximity sensor based on magnetic force for hydrogenerator applications", presented at Proc. IASTED International Conference, Crete, Greece, 2008.

[9] G. P. Agrawal, Fiber Optics Communiction Systems. New York: John Wiley \& Sons, 1992.

[10] A. D. Kersey, "A review of recent developments in fiber optic sensor technology, fiber optic smart structures," U. S. Naval Research Laboratory, Washington, February 13, 1996.

[11] T. Hiroshi, K. Kenji, and O. Shinji, "Ultrasonic sensitivity of strain-insensitive fiber Bragg grating sensors and evaluation of ultrasound-induced strain," Sensors, vol. 10, no. 12, pp. 11248-11258, 2010. 